\title{
Three-dimensional analysis by a microlens-array confocal arrangement
}

\author{
Hans J. Tiziani and Hans-Martin Uhde
}

\begin{abstract}
Scanning confocal microscopy is now well developed and applied. As an alternative to a laser spot to be scanned, parallel processing can be obtained when a two-dimensional structure is moved through the focal plane and a series of image sections is recorded. Surface topography is determined by analysis of the normalized intensity of the appropriate image points, i.e., a search of the intensity maximum leads to surface coordinates. With a high numerical aperture of the optical system, the half-width of $I(z)$ is small, and the topography can be calculated with high accuracy. But with a high numerical aperture, only small object fields can be reproduced. As an alternative to the Nipkow disk for parallel processing, high-numerical-aperture microlenses are combined in an array. The reproducible object field is then limited by the size of the array and the number of lens and detector elements.

Key words: Three-dimensional analysis, confocal microscopy, microlens array, parallel image processing.
\end{abstract}

\section{Introduction}

There are different techniques of measuring surface topography that are based on, e.g., triangulation, projected fringes, interferometry, and holography. In addition, the confocal principle is also suitable, as it leads to a true depth discrimination. ${ }^{1-3}$ For the confocal scanning principle, those parts of the object that are located in the focus appear sharp and bright in the corresponding image plane, whereas parts that are outside the focus appear blurred and dark. Even for phase objects with no amplitude variations, the three-dimensional (3-D) structure can be analyzed.

High lateral and depth resolutions can be obtained with the confocal principle when high-numericalaperture objectives are used. Furthermore, for parallel processing, diffraction-limited two-dimensional spot arrays can be projected onto the object, as first proposed by Petran et al. ${ }^{4}$ and Xiao et al. ${ }^{5}$ They used a Nipkow disk to generate the pinholes. For high spatial and depth resolutions, a high-numericalaperture lens must be used for the projection of light spots onto the object. The price to pay for a highly magnifying microscope objective with very high numerical aperture $(>0.8)$ is a low field size.

The authors are with Institut für Technische Optik der Universität Stuttgart, Pfaffenwaldring 9, 70569 Stuttgart, Germany.

Received 20 May 1993; revision received 27 July 1993.

0003-6935/94/040567-06\$06.00/0.

- 1994 Optical Society of America.
The alternative to using low-power objectives with low numerical apertures leads not only to a larger field size but also to a moderate depth discrimination. The theoretical axial focus shift necessary at the 3-dB point, where the intensity is reduced to its half value, is $\sim 0.36 \mu \mathrm{m}$ when a $160 \times / 0.95$ objective is used at a wavelength of $546 \mathrm{~nm}$ and $\sim 2.3 \mu \mathrm{m}$ when a $20 \times / 0.45$ objective is used. An alternative is a microlens array, which is described below.

For the contouring of large object fields with high depth discrimination, microlens arrays, in which each microlens has a high numerical aperture, are suitable. A large number of microlenses can be considered for a large object field without a reduction in the depth discrimination. The numerical aperture and the object field are no longer directly coupled.

When a microlens array is used, the numerical aperture is defined by the size of the individual lenses, and the object field is defined by the size of the array and the number of detector elements. We give some options to extend the application of the principle of confocal microscopy to the topography of some technical surfaces in mechanical engineering for surface characterization, for instance.

\section{Whole-Field Confocal Principle}

The principle of the reflection-type whole-field confocal imaging system is indicated in Fig. 1. In the basic arrangement of a reflection confocal microscope a point light source is considered at pinhole $\mathrm{P}$ to be reproduced by the objective at a diffraction-limited 


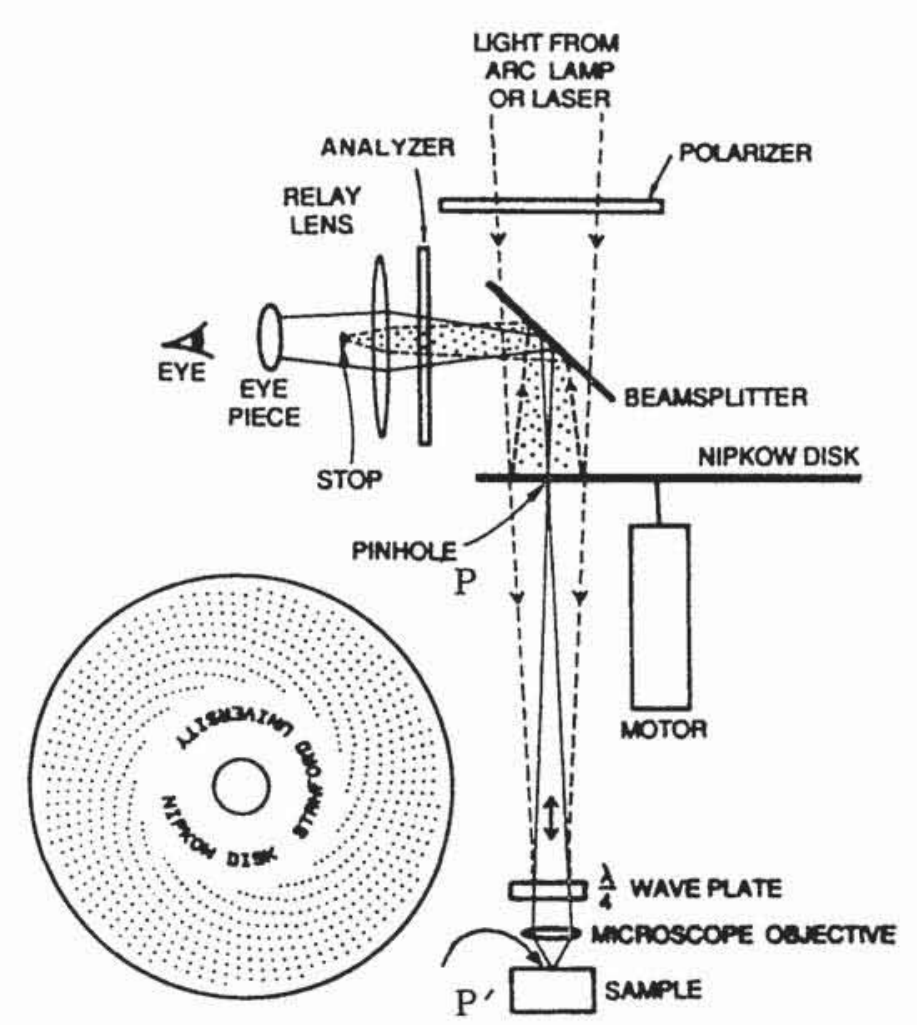

Fig. 1. Principle of a whole-field confocal microscope. ${ }^{6}$

point $\mathrm{P}^{\prime}$ on the object. The reflected light at $\mathrm{P}^{\prime}$ is collected by the same objective, focused to the pinhole, and imaged to the detector. In perfect autocollimation the detector signal has its maximum. If the object point is shifted axially, the incoming light is distributed on an object area around $\mathrm{P}^{\prime}$. The light flux passing through the pinhole at $\mathrm{P}$ is reduced. Therefore one can reduce the detector signal by shifting the object axially. The intensity distribution in the $z$ direction can be approximately written, according to Ref. 6 , in the pseudoparaxial region as

$$
I(z)=\left\{\frac{\sin [k z(1-\cos \alpha)]}{k z(1-\cos \alpha)}\right\}^{2},
$$

where $k$ is the wave number, and $k=2 \pi / \lambda$, where $\lambda$ is the wavelength and $\sin (\alpha)$ is the numerical aperture, NA, of the image-forming objective.

In Eq. (1) the intensity drops to its half-width for $k z(1-\cos \alpha) \simeq 1.39$. For the full width at halfmaximum (FWHM) we find

$$
\mathrm{FWHM}=\frac{0.44 \lambda}{1-\cos \alpha} .
$$

By axially shifting the object and observing the intensity at the detector, we can determine the $z$ coordinate of $\mathrm{P}^{\prime}$ with high accuracy. To analyze the whole object, we must scan the light spot over the object area. Alternatively, a rotating Nipkow disk can be used, as shown in Fig. 1. It was first proposed by Petran et al. in $1968^{4}$ and in a modified version by Xiao et al. in 1988. ${ }^{5}$ Several points on the object are now illuminated simultaneously; the light spots should be diffraction limited and the distance between the holes on the disk must be chosen appropriately to avoid an overlap of the spot images. For focused light spots, the system works in autocollimation. Furthermore, light passing one hole should not contribute, when returning, to a neighboring hole in order to avoid a reduction in depth discrimination. In the detector plane, a CCD camera can be used for image spot detection; in addition, an eyepiece is used for visual observation. If the Nipkow disk is rotating fast enough, a complete image of the object can be observed where perfectly conjugated object points passing the Nipkow disk appear bright; out-of-focus points are darker. Focusing through the object topography leads to a set of height sections of the object.

Great care needs to be taken to avoid stray light and light reflected by the Nipkow disk and other optical components to reach the detector, such as the CCD camera. Because the surface of the disk can be strongly reflecting and if the ratio between diameter and distances of the holes is $1: 10$, the ratio of light returned from even strong reflecting objects to be received by the detector is weak compared with that reflected by the disk. In the arrangement that we used, the spot size on the disk was $20 \mu \mathrm{m}$. With the necessary precautions taken (tilted Nipkow disk, spatial filtering, and polarization), the system works well.

In Figs. 2 and 3, standard surfaces calibrated at the Physikalisch-Technische Bundesanstalt (PTB) in Braunschweig, Germany, which were analyzed with our modified experimental setup depicted in Fig. 1, are shown.

First we present results obtained from a PTB calibration standard, which includes six grooves with groove depths between 9 and $0.251 \mu \mathrm{m}$. The interferometrically obtained calibration measurement of the PTB gave a depth of $(5.66 \pm 0.05) \mu \mathrm{m}$ for the second groove. Results of this groove, obtained with our developed confocal microscope depicted in Fig. 1, are shown as a 3-D plot in Fig. 2(a) and as a profile in Fig. 2(b), which gives a depth of $\sim 5.70 \mu \mathrm{m}$.

Figure 3 shows results of measured profiles obtained from a roughness standard that was calibrated at the PTB. This standard shows roughness with a lateral periodicity of $4 \mathrm{~mm}$, a maximum roughness depth $R_{\max }$ of $9.60 \mu \mathrm{m}( \pm 4 \%)$, and a mean roughness depth $R_{a}$ of $1.45 \mu \mathrm{m}$ ( $\left.\pm 4 \%\right)$ with a cutoff wavelength $\lambda_{c}$ of $0.8 \mathrm{~mm}$. In Fig. 3(a) the PTB-calibrated stylus profile record is shown. The profile within section $A-A^{\prime}$ of Fig. 3(a) was measured with our confocal microscope (Fig. 1) and is shown in Fig. 3(b). It was obtained by four measurements laterally displaced by one field of view and combined afterward. The vertical displacement of the four measurements of Fig. $3(b)$ is due to runout errors of the lateral positioning device of our microscope; therefore it is insignificant. A very close agreement of the microprofile between the results of both measuring methods can be seen. 

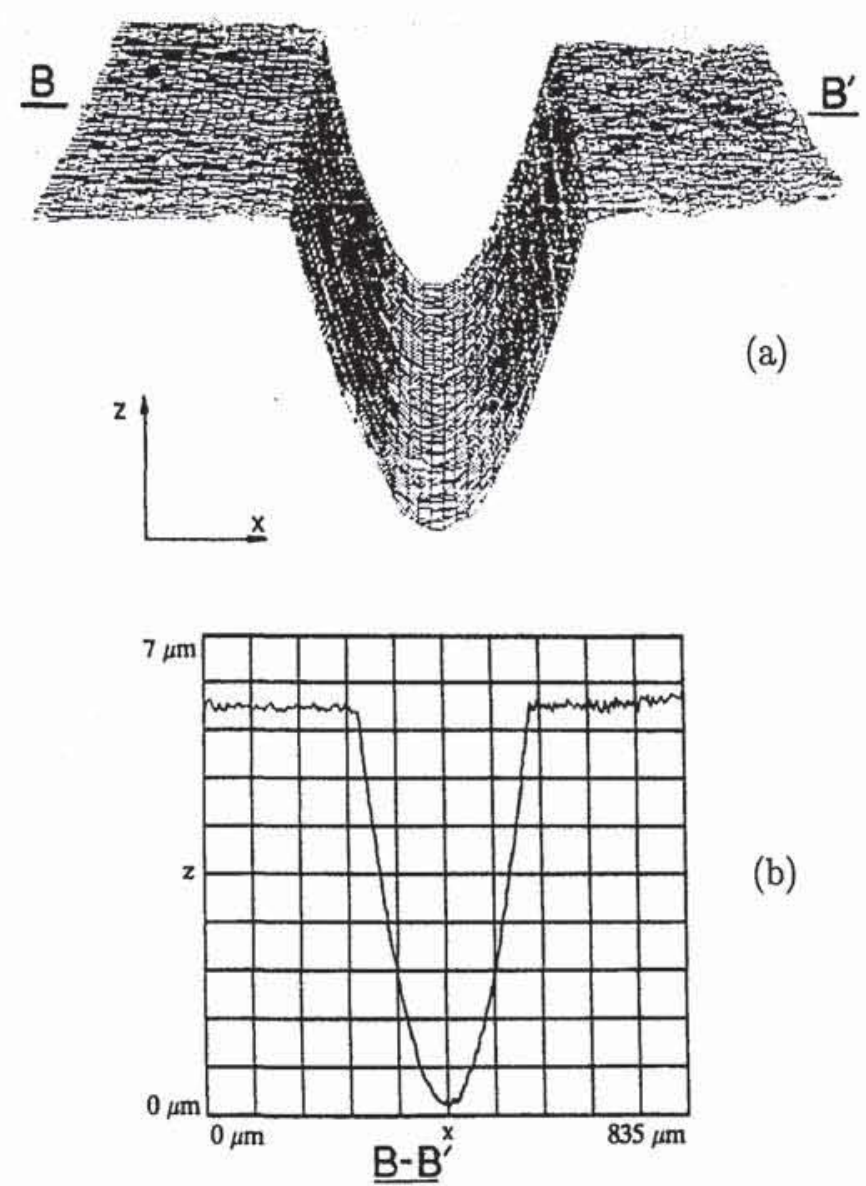

Fig. 2. (a) Confocal measured 3-D plot of one groove of the calibration standard. (b) Profile $B-B^{\prime}$ of the 3-D plot of Fig. 2(a). The groove depth of the PTB calibration measurement is $(5.66 \pm 0.05) \mu \mathrm{m}$; the depth measured with our confocal setup is $5.70 \mu \mathrm{m}$.

To improve the light efficiency and extend the field of view without losing depth discrimination, a modified principle based on microlenses is described in Section 3.

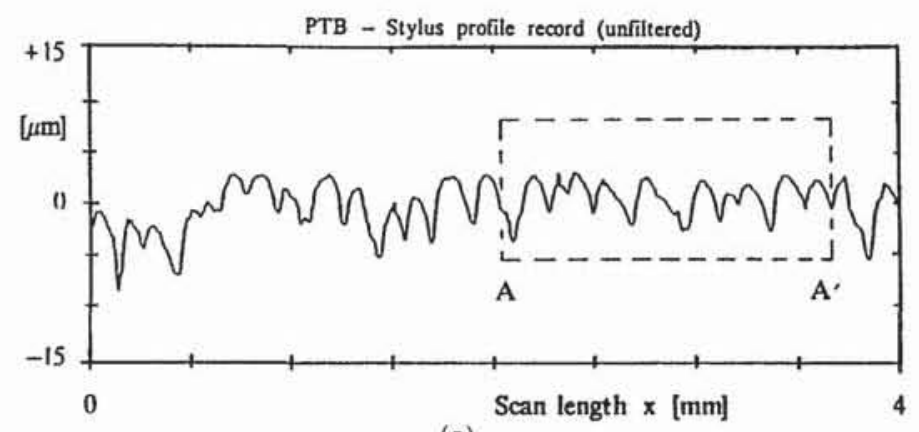

(a)

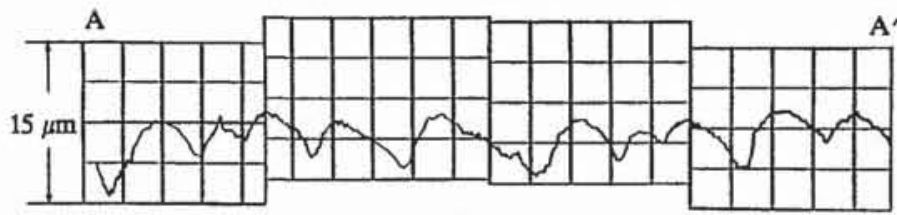

(b)

Fig. 3. (a) Stylus profile record of a roughness standard calibrated at the PTB. Section $A-A^{\prime}$, with a lateral distance of $\sim 1.6 \mathrm{~mm}$, is marked. (b) Profile of the marked section, $A-A^{\prime}$, measured with the confocal principle of Fig. 1.

\section{Microlens-Array Arrangement}

To extend the object field and to improve the light efficiency of the above-described confocal system by using the Nipkow disk or other appropriate masks, we used a microlens array. In Figure 4 our experimental system for 3-D analysis with a microlens array, in which as many as $300 \times 300$ elements were used, is shown. Light coming from a laser or, alternatively, from a xenon arc lamp is focused by the condenser lens system to a pinhole. The light passing the pinhole is collimated by lens L2. The parallel beam, as shown schematically in Fig. 4, is focused by the microlenses to a two-dimensional point array. The microlenses focus the spots nearly into a plane. For high depth discrimination, the focal distance of the microlenses must be short in order to work with a high numerical aperture. The numerical aperture of the microlenses used was 0.26 with a focal length of $f_{4}{ }^{\prime}=230 \mu \mathrm{m}$. A parallel beam is formed from object points in focus when passing the microlenses a second time. The light spots reflected from the object points in focus are formed at infinity and focused by lens L3 to a small spot at a distance $f_{3}{ }^{\prime}$ (the focal distance of L3). After passing a spatial filter in the back focal plane, the light reaches the CCD chip of the camera, where the object is imaged. The image of the camera is processed by a computer with a frame grabber. This image is digitized and stored in memory. It should be noted that the object topography is digitized by the spot separation, which was $125 \mu \mathrm{m}$ in this particular setup. The lateral resolution is given by the spot diameter of the microlenses, which is of the order of $3 \mu \mathrm{m}$. For some applications it may be useful to reduce the separation of the sampling spots by shifting the surface under test laterally by a fraction of the microlens separations.

The depth discrimination arises in a manner similar to conventional confocal microscopy: If the object is moved out of the focal plane, the single points of the two-dimensional point array are no longer in autocollimation. A small portion of the light passes the pinhole, and a reduced amount of light flux reaches the detector elements.

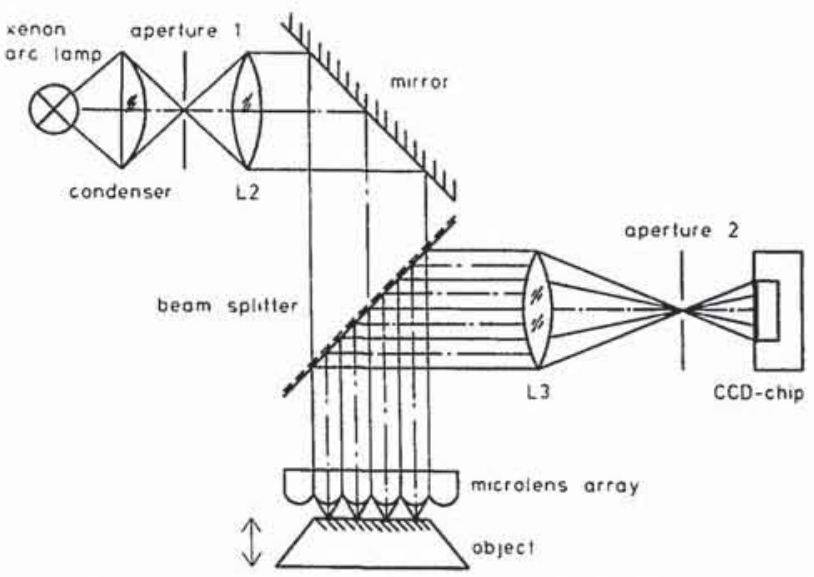

Fig. 4. Arrangement for confocal 3-D analysis with a microlens array. 
The microlenses were generated on a glass substrate, which led to some back reflection by the upper side of the glass plate. A slightly tilted plate avoids the reflection light to pass the second aperture and reach the camera.

The main advantages of a confocal system with a microlens array are greater light efficiency compared with the arrangement with the Nipkow disk, namely, by a factor of nearly 100 , and the large object field. Nevertheless there are also some drawbacks for some applications, namely, the relatively small working distance.

\section{Three-Dimensional Analysis with a Microlens Array}

For analysis of the 3-D topography of an object, the object or the microlenses are moved stepwise in axial direction. At each position an image is stored in the frame grabber. A series of 256 height sections with, for instance, the following intensity distributions

$$
I(i, j ; k), i=1 \cdots 512, j=1 \cdots 512, k=1 \cdots 256,
$$

can be stored. The index numbers $i$ and $j$ are the pixel numbers in the digitized images in the $x$ and $y$ directions given by the light spots, and $k$ is the

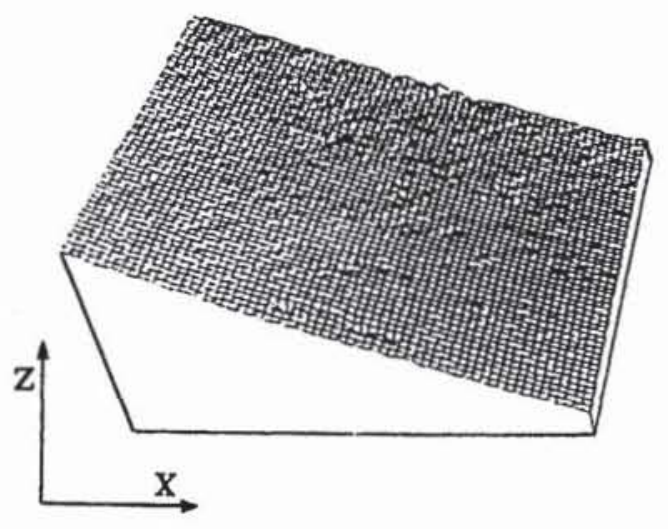

(a)

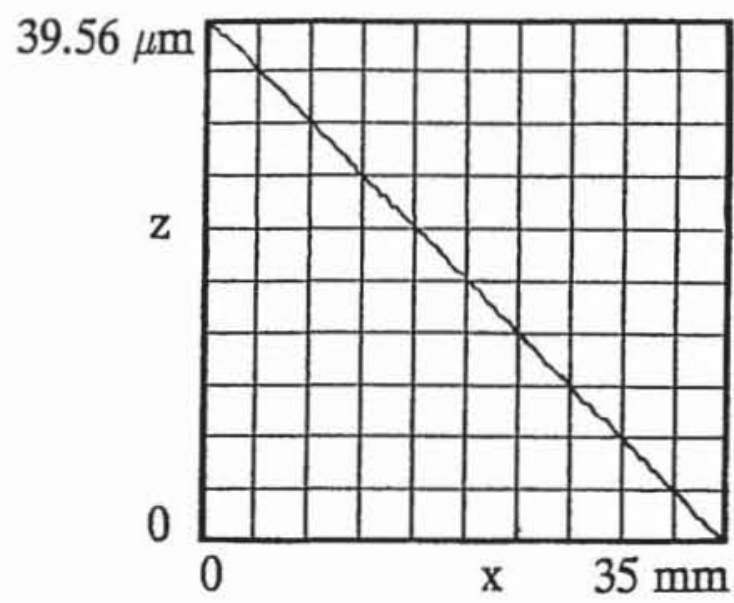

(b)

Fig. 5. (a) 3-D plot of a tilted mirror surface $35 \mathrm{~mm} \times 35 \mathrm{~mm}$ and a total height variation of $39.56 \mu \mathrm{m}$. (b) Section of this surface. number of the height sections. The axial position of the object at one height section is $k \mathrm{~d} z$. In the search for the brightest pixel, the height of the object point represented by pixel $(i, j)$ is obtained. If $\mathrm{k}$ is the number of this section, the height of the object point is $z=\kappa \mathrm{d} z$.

For the whole series of images, we get the data set

$$
\text { к(i,j) } \begin{aligned}
\text { where } i & =1 \cdots 512, \\
j & =1 \cdots 512, \\
1 & \leq \kappa \leq 256,
\end{aligned}
$$

which can be interpreted as an 8-bit-deep image. This image is shown on the monitor. The intensity of each pixel $(i, j)$ gives its $\mathrm{\kappa}$ value and from that, regardless of the distance of the sections $\mathrm{d} z$, the height of the object point belonging to pixel $(i, j)$. The matrix of the brightest pixels $\kappa(i, j)$ leads to the topography of the scene.

As additional information, the relative reflectivity of each digitized object point can be recorded from the autofocus picture synthesized out of the 256 height sections:

$$
A(i, j)=I[i, j ; \kappa(i, j)] .
$$

The result can be presented in different ways. For example, information about the autofocus image $A(i, j)$

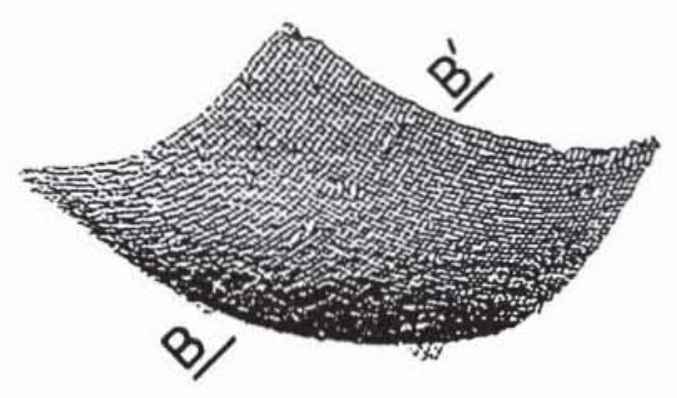

(a)

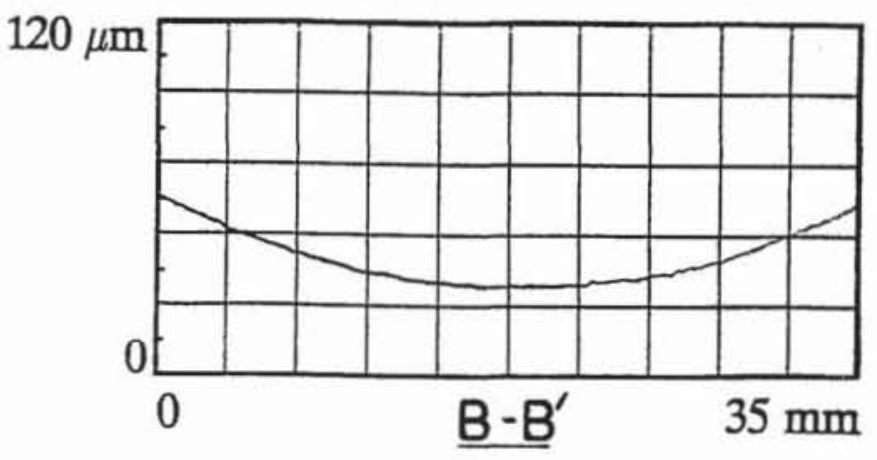

(b)

Fig. 6. (a) $3-D$ plot of a spherical surface $35 \mathrm{~mm} \times 35 \mathrm{~mm}$ with a radius of curvature of $4000 \mathrm{~mm}$ and a height resolution of 90 $\mathrm{nm}$. (b) Cross section of this profile. 
and the height image $\kappa(i, j)$ can be combined to generate stereoscopic images or anaglyphic images to be looked at with red-green glasses.

Care needs to be taken for the nontransparent parts of the microlens array between the hexagonally arranged lenses to avoid light reaching the detector, which could lead to some noise and eventually to height errors.

One can obtain an improved height determination compared with the data set of the brightest pixels $\kappa(i, j)$ by taking the center of gravity $(\operatorname{cog})$ of this intensity series:

$$
\kappa_{\operatorname{cog}}(i, j)=\frac{\sum_{k=1}^{256} k I(i, j ; k)}{\sum_{k=1}^{256} I(i, j ; k)}
$$

Errors arising from the different focal distances for different microlenses can be eliminated by calibration of the system with a perfect mirror surface, which is stored as reference. This structure must be subtracted from each measured topography of an object.

\section{Experimental Results}

A laser as well as a white-light source were used in the experimental setup, as shown schematically in Fig. 4. A xenon arc lamp CSX 75W/2 from Philips, which gives a continuous spectrum similar to natural daylight in the visible range, was used in the experiments reported here. To reduce the influence of the chromatic aberration, a narrow-band color filter was used for some experiments. Different microlens arrays produced with different techniques were used. One element, which was fabricated on a photoresist, was kindly provided by Hutley. ${ }^{7}$ The diameter of the lenses of the two-dimensional array used in the following experiments was $125 \mu \mathrm{m}$; the lenses were arranged hexagonally over an area of $70 \mathrm{~mm} \times 70$ $\mathrm{mm}$. The mean focal length was $230 \mu \mathrm{m}$, and the numerical aperture was 0.26 . A microscope stage was used for the basic setup. The height shift in the first setup was obtained by a stepper motor, which (a)

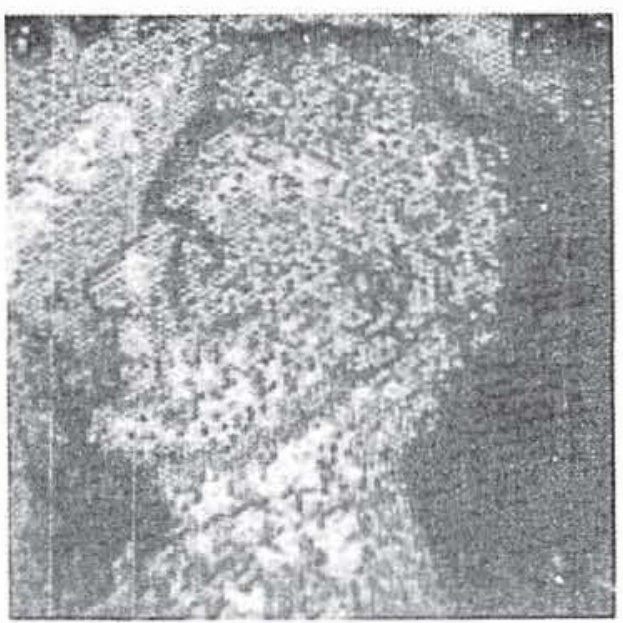

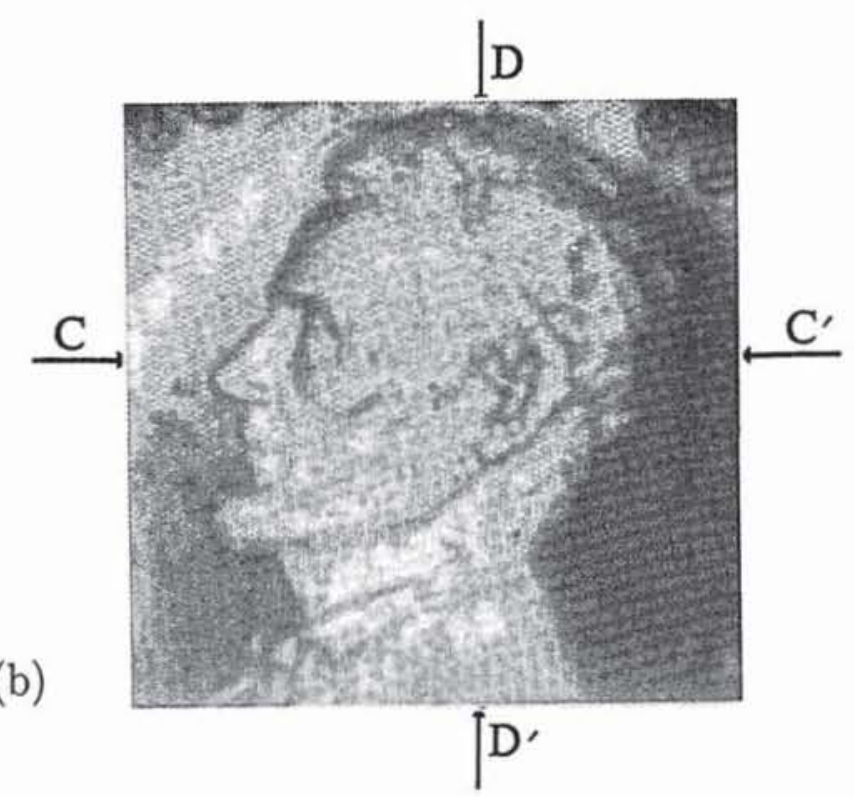

154

(d)

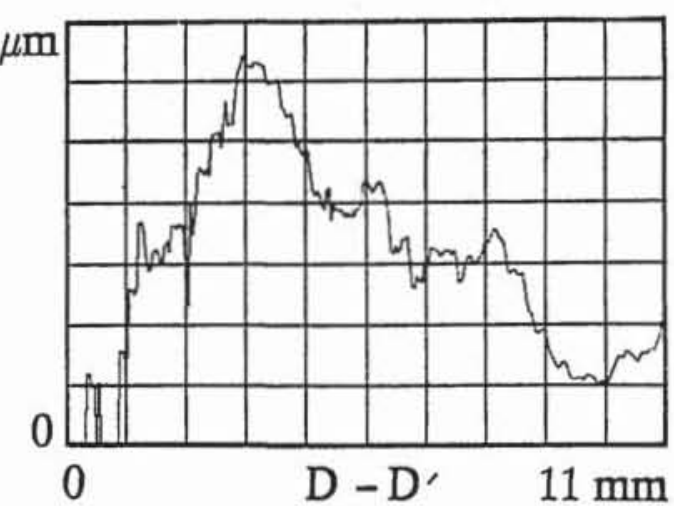

154

(c)

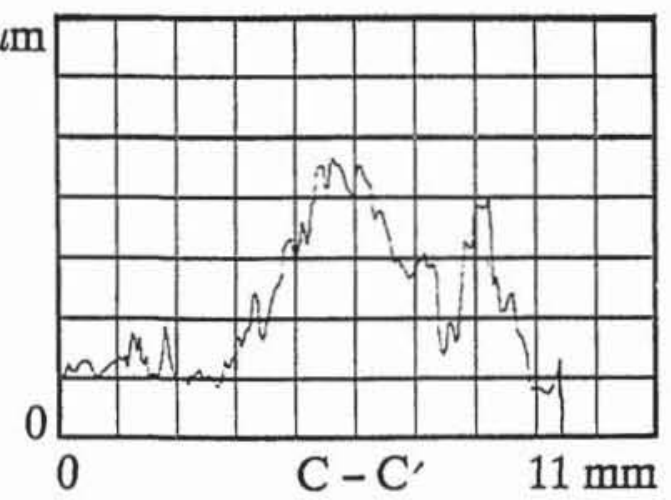

Fig. 7. Analysis of a portion of a one-cent piece: field size $11 \mathrm{~mm} \times 11 \mathrm{~mm}$. The spot separations were (a) $125 \mu \mathrm{m},(\mathrm{b}) 125 \mu \mathrm{m} / 4=31.25$ $\mu \mathrm{m}$ when the sample was shifted. (c), (d), Cross sections of (b), perpendicular to each other. 
allowed axial shifts of the object in steps of $0.5 \mu \mathrm{m}$. The distance $d z$ between two sections can, however, be adapted to the need and can be chosen as a multiple of $0.5 \mu \mathrm{m}$. A Sony CCD camera with $512 \times$ 512 pixels together with a low-noise amplifier were used as the detector. The first computer used was a Vicom with a 2-Mbyte image memory, but we now use an IBM-compatible 486 computer with frame grabber. From the object to the image, plane magnifications of $\beta^{\prime}=-0.2$ for Figs. 5 and 6 and -0.6 for Fig. 7 were used to form the image on a CCD chip. With a focal length for lens L3 of $f_{3}{ }^{\prime}=100 \mathrm{~mm}$, and by using a pinhole, we found that a tilt of the back surface of the microlens array of $0.3^{\circ}$ was sufficient to avoid stray light from the back surface.

For the height evaluation of the following examples, 256 steps in the $z$ direction were used; the center-of-gravity method was used for higher depth resolution. At first the dependency of the intensity $I(z)$ was measured with a tilted perfect mirror as a reference object to determine the quality of the depth discrimination (Fig. 5).

The measured FWHM was $25 \mu \mathrm{m}$, even though the theortical FWHM at wavelength $\lambda=546 \mathrm{~nm}$, according to Eq. (2), was $7 \mu \mathrm{m}$. Because there are residual aberrations such as spherical aberrations and broadband illumination, the experimental FWHM was broadened to $25 \mu \mathrm{m}$. The depth resolution was 0.09 $\mu \mathrm{m}$, and the field size was $35 \mathrm{~mm} \times 35 \mathrm{~mm}$.

In Fig. 6 the plot of a spherical mirror that was analyzed with a radius of curvature of $4000 \mathrm{~mm}$ is shown. The 3-D plot presentation is shown in Fig. 6(a), and a cross section is shown in Fig. 6(b). The field size was again $35 \mathrm{~mm} \times 35 \mathrm{~mm}$.

The topography of an one-cent piece is shown in Fig. 7. The field size was $11 \mathrm{~mm} \times 11 \mathrm{~mm}$. In Fig. $7(\mathrm{a})$, the separation of the spots was $125 \mu \mathrm{m}$, whereas in Fig. 7(b) the spot separations were $31.25 \mu \mathrm{m}$ because of lateral shifts of the one-cent piece in quarters of the $125-\mu \mathrm{m}$ separation of the microlens elements. An improvement of the lateral resolution can be seen. In Figs. 7(c) and 7(d) two cross sections of Fig. 7(b) are shown; they were selected to be perpendicular to each other. The peak-to-valley height was $132 \mu \mathrm{m}$.

The measuring time depended on the number of height sections considered. There is a trade-off between the depth resolution $d z$ of the system and the measuring time. The center-of-gravity method, as described by Eq. (4), leads to the best height determination.
The resolution is increased, on the average, by a factor of $\sqrt{2}$ when the number of height sections is increased by a factor of 2 . This agrees with the theory. ${ }^{8}$

\section{Conclusion}

A confocal measuring system based on microlens arrays was developed for the topometry and surface characterization of technical surfaces. The method was applied to examine flat structures with a lateral area of $40 \mathrm{~mm} \times 40 \mathrm{~mm}$ and height resolutions of 90 $\mathrm{nm}$. The working distance is limited because the focal length of the microlenses was $230 \mu \mathrm{m}$. Although there are some limitations, there are interesting applications, especially in mechanical engineering for surface characterization and in microelectronics. Large object fields can be analyzed in a short time with high depth discrimination. Improving the quality of the microlenses can increase the performance.

The financial support of the Deutsche Forschungsgemeinschaft is gratefully acknowledged. Furthermore, we thank R. Achi and H.-J. Jordan for providing some experimental support.

\section{References}

1. T. Wilson and C. J. R. Sheppard, Theory and Practice of Scanning Optical Microscopy (Academic, London, 1984), Chaps. 1,2 , and 4.

2. G. J. Brakenhoff, P. Blom, and P. Barends, "Confocal scanning light microscopy with high aperture immersion lenses," J. Microsc. 117, 219-232 (1979).

3. K. Carlsson and N. Aslund, "Confocal imaging for 3-D digital microscopy," Appl. Opt. 26, 3232-3238 (1987).

4. M. Petran, M. Hadravsky, M. D. Egger, and R. Galambos, "Tandem-scanning reflected-light microscope," J. Opt. Soc. Am. 58, 661-664 (1968).

5. G. Q. Xiao, T. R. Corle, and G. S. Kino, "Real time confocal scanning optical microscope,” Appl. Phys. Lett. 53, 716-718 (1988).

6. C. J. R. Sheppard and H. J. Matthews, "The extended-focus, auto-focus and surface-profiling techniques of confocal microscopy," J. Mod. Opt. 35, 145-154 (1988).

7. M. C. Hutley, "The manufacture and testing of microlens arrays," in Optics in Complex Systems, F. Lanzl, H. Preuss, and G. Weigelt, eds., Proc. Soc. Photo-Opt. Instrum. Eng. 1319, 491-492 (1990).

8. G. Zinser, U. Harbarth, and H. Schröder, "Formation and analysis of three-dimensional data with the laser tomographic scanner (LTS)" in Scanning Ophthalmoscopy and Tomography, J. E. Nasemann and R. E. Burk, eds. (Quintessenz-Verlag, Berlin, 1990), pp. 243-252. 